\title{
Risk Factors for Adrenal Crisis in Patients with Adrenal Insufficiency
}

\author{
KAZUE OMORI, KAORU NOMURA, SATORU SHIMIZU*, NARIKO OMORI AND KAZUE TAKANO \\ Department of Medicine, Institute of Clinical Endocrinology, Tokyo Women's Medical University, Shinjuku-ku, Tokyo 162-8666 \\ Japan \\ *Department of Hygiene and Public Health I, Tokyo Women's Medical University, Shinjuku-ku, Tokyo 162-8666 Japan
}

\begin{abstract}
Patients with adrenal insufficiency have a life-threatening risk of adrenal crisis, thus preventing adrenal crisis is an important clinical issue. In order to clarify the risk factors for adrenal crisis, the medical records of 137 patients with established adrenal insufficiency were retrospectively investigated. The explanatory variables analyzed were gender, etiology of hypoadrenalism, class of adrenocortical hormone replaced, duration of steroid replacement, age at time of survey, age at time of diagnosis of hypoadrenalism, state of other hormone deficiencies (growth hormone and sex steroids), diabetes insipidus, and mental disorder. Diagnosis of adrenal crisis was based on physical and laboratory findings. Forty (29\%) of the 137 patients had at least one episode of adrenal crisis. Based on the Akaike Information Criterion (AIC), steroid replacement therapy of more than 4 yrs' duration was the largest single contributor to the occurrence of an adrenal crisis, followed by mental disorder and sex steroid deficiency. In the subclass of patients with secondary adrenal insufficiency $(\mathrm{N}=115)$, sex steroid deficiency was the greatest risk factor. Patients with untreated hypogonadism had a significantly higher relative risk of 3.70 (95\% confidential interval: 1.71-7.98) compared to those without hypogonadism or with treated hypogonadism. Furthermore, among patients with hypogonadism aged younger than $50 \mathrm{yrs}$, those treated with sex hormone (5/51: 10\%) suffered less frequently from adrenal crisis than those untreated ( $7 / 11: 64 \%, p=0.0004)$. In conclusion, the present study has, for the first time, clarified the risk factors of adrenal crisis. Among them, sex hormone deficiency has an especially important implication because it can be treated by hormone replacement therapy with the hope of reducing the risk of adrenal crisis.
\end{abstract}

Key words: Adrenal insufficiency, Adrenal crisis, Hypogonadism, Hormone replacement therapy

(Endocrine Journal 50: 745-752, 2003)

GLUCOCORTICOIDS are normally secreted from the adrenal cortex in large quantities during physiological stress in order to maintain homeostasis. However, in patients with adrenal insufficiency, adrenal crisis presents a life-threatening risk in response to stress such as infection, major illness, and the inability to retain medication.

According to research carried out in Japan, the prevalence of adrenal crisis is $9.5 \%$ in patients with Addison's disease in a period of 3 to $15 \mathrm{yrs}$ [1], 10.4\%

Received: March 31, 2003

Accepted: August 11, 2003

Correspondence to: Dr. Kazue OMORI, Department of Medicine, Institute of Clinical Endocrinology, Tokyo Women's Medical University, Kawada-cho 8-1, Shinjuku-ku, Tokyo 162-8666, Japan in patients with secondary adrenal insufficiency in a period of 3 to $15 \mathrm{yrs}$, and $21 \%$ in a period of 1 to $35 \mathrm{yrs}$ [2]. Braatvert et al. from a questionnaire survey reported that about half of their 25 patients with Addison's disease have experienced adrenal crisis [3]. Although early symptoms of adrenal crisis are nonspecific, shock can progress to coma and death without appropriate therapy, hence adrenal crisis requires immediate diagnosis and appropriate treatment. In order to prevent an adrenal crisis, the patient's own awareness and that of responsible family members regarding the condition is important. They must be taught about the need to increase their daily dosage or to seek medical assistance and to carry their Emergency Medical Information Card. However, patient knowledge is not enough under conditions of physio- 
logical stress. Besides effective lifelong education $[4,5]$, it is important to clarify the risk factors underlying the occurrence of an adrenal crisis in order to prevent it. However, the risk factors for adrenal crises have never been reported. In this study, we retrospectively analyzed the background of patients in an attempt to clarify the risk factors for adrenal crisis.

\section{Subjects and Methods}

\section{Subjects}

The medical records of 137 patients with established adrenal insufficiency were investigated retrospectively. These patients had medical consultation at our institute from January 1990 to April 2000. Five patients had died in the course of this term, but it was unclear whether the causes of death were related to adrenal crisis.

We diagnosed the patients who were receiving replacement steroids as having adrenal insufficiency. The different causes that contributed to primary and secondary adrenal insufficiency are summarized in Table 1. Of patients with primary adrenal insufficiency, 8 cases were congenital adrenal hyperplasia (one male 17-OHD and seven female 21-OHD). As to seven females with 21-OHD, five cases had normal or low serum DHEA and urinary 17-KS levels under

Table 1. Patients with primary and secondary adrenal insufficiency

\begin{tabular}{lrrr}
\hline & No & male & female \\
\hline Primary & 22 & 8 & 14 \\
$\quad$ Addison's disease & 11 & 6 & 5 \\
Congenital adrenal hyperplasia & 8 & 1 & 7 \\
Bilateral adrenolectomy & 3 & 1 & 2 \\
\hline Secondary & 115 & 53 & 62 \\
$\quad$ Panhypopituitarism after surgery & 76 & 35 & 41 \\
$\quad$ and/or radiation* & & & \\
Isolated ACTH deficiency & 3 & 3 & 0 \\
Idiopathic hypopituitarism & 14 & 12 & 2 \\
Empty sella & 7 & 3 & 4 \\
Sheehan's syndrome & 15 & 0 & 15 \\
\hline Total & 137 & 61 & 76
\end{tabular}

*Original diagnoses were pituitary non-functioning adenoma (22), prolactinoma (7), Cushing's disease (7), acromegaly (3), Rathke's cleft cyst (1), craniopharyngioma (17), and germinoma (19). steroid replacement. Their serum testosterone levels were all less than $5 \mathrm{ng} / \mathrm{dl}$. Three of them were being treated by sex hormone replacement therapy. Two other cases with 21-OHD were postmenopausal age and their androgen levels were presumed to be low. Three cases of primary adrenal insufficiency were complicated with primary hypothyroidism.

All patients were on replacement steroid therapy, glucocorticoid (G) and/or mineralocorticoid (M). Hydrocortisone has both $\mathrm{G}$ and $\mathrm{M}$ activity, prednisolone and dexamethasone have $\mathrm{G}$ activity, and fludrocortisone has $\mathrm{M}$ activity. The daily dosage of steroid replacement was as follows: $5-20 \mathrm{mg}$ hydrocortisone $(\mathrm{N}=119), 10 \mathrm{mg}$ hydrocortisone with $0.1-1 \mathrm{mg}$ dexamethasone $(\mathrm{N}=9)$, or $0.1-0.375 \mathrm{mg}$ dexamethasone with $0.05 \mathrm{mg}$ fludrocortisone $(\mathrm{N}=2)$ as $\mathrm{G}+\mathrm{M}, 5 \mathrm{mg}$ prednisolone $(\mathrm{N}=2)$ or $0.4-1 \mathrm{mg}$ dexamethasone $(\mathrm{N}=$ 5 ) as $\mathrm{G}$. The replacement periods ranged from 1 to 35 yrs. As regards patients treated with over $20 \mathrm{mg}$ hydrocortisone or $0.5 \mathrm{mg}$ dexamethasone, the reason why they needed such high quantities of steroid was that certain symptoms such as general fatigue appeared and their ACTH level was high. The diagnosis of adrenal crisis has yet to be defined. The present study defined an adrenal crisis as a patient presenting with hyponatremia $(\leq 134 \mathrm{mEq} / 1, \mathrm{~N}=29)$, hypoglycemia $(\leq 50 \mathrm{mg} / \mathrm{dl}, \mathrm{N}=3$ ), hypotension (systolic blood pressure $\leq 80 \mathrm{mmHg}, \mathrm{N}=6$ ), or unconsciousness (clouding of consciousness, $\mathrm{N}=29$ ), when there was any suspicion of adrenal crisis from clinical signs and symptoms. In cases that have experienced plural crises, we used the data from the first crisis.

We diagnosed growth hormone (GH) deficiency based on the standard of diagnosis which was published in 1988 and has been recognized worldwide [6], namely, an insulin tolerance test that showed a GH peak of under $3 \mathrm{ng} / \mathrm{ml}(\mathrm{N}=33)$. If this standard was not met, one of the following conditions was needed: a) there was an organic abnormality of the hypothalamus and/or pituitary and there was at least one additional pituitary hormone deficiency besides ACTH, and an arginine or GRF test that showed a GH peak under $3 \mathrm{ng} / \mathrm{ml}(\mathrm{N}=23)$; or b) the serum IGF-1 concentration was under $2 \mathrm{SD}$ of the age standard $(\mathrm{N}=32)$. Six patients had GH deficiency but were on GH replacement therapy almost throughout the entire period of this study, and were thus not regarded as having any GH deficiency.

The diagnosis of sex hormone deficiency was amen- 
orrhea in females $(\mathrm{N}=42)$ and low serum testosterone in males $(\mathrm{N}=9)$, or the responses of LH and FSH were low by LH-RH in case of no testosterone data $(\mathrm{N}=1)$. We did not regard patients who were being treated by hormone replacement therapy such as estrogen and progesterone $(\mathrm{N}=30)$ or testosterone enanthate $(\mathrm{N}=$ 40) as having sex hormone deficiency. The period of replacement was between one to $26 \mathrm{yrs}$, and three patients had stopped replacement therapy for from six months to three yrs, or had been treated intermittently, but we regarded them as not having any deficiency because their replacement terms were long.

We diagnosed diabetes insipidus as replacement with desmopressin acetate $(\mathrm{N}=48)$ after pituitary operation and/or radiation.

We also diagnosed hypothyroidism due to hypopituitarism as treatment with levothyroxine after pituitary operation and/or radiation. The number of hypothyroidism patients was 105 and the daily dose of levothyroxine was 25 to $200 \mu \mathrm{g}$, with replacement periods ranging from one to $28 \mathrm{yrs}$.

We diagnosed patients under psychiatric consultation and those with obvious procephalic impairment and dementia after a pituitary operation as having a mental disorder.

\section{Items of investigation}

In order to clarify the risk factors for the occurrence of an adrenal crisis, we analyzed the following explanatory variables: age at time of survey, age at time of diagnosis of hypoadrenalism, gender, etiology of hypoadrenalism (primary/secondary), class of adrenocortical hormone replaced $(G$ or $G+M)$, duration of steroid replacement, state of other hormone deficiencies (GH and sex steroids), presence of diabetes insipidus, and presence of any mental disorder (depression, poor intelligence, procephalic impairment and dementia after an operation).

\section{Statistical analysis}

We used the Akaike Information Criterion (AIC) [7, 8] to statistically evaluate how the above-mentioned items contributed to an adrenal crisis. In other words, the presence of an adrenal crisis was the objective variable and the above-mentioned items which were purported the risk factors were the explanatory variables. We then extracted the information that each explanatory variable gave to the objective variable. The statistics of the AIC show how the explanatory variable affects the objective variable. The lower the AIC value, the more explanatory it is. Each explanatory variable was divided into two groups, namely, the gender was male/female, the etiology of hypoadrenalism was primary/secondary, the class of adrenocortical hormone was $\mathrm{G}+\mathrm{M} / \mathrm{G}$, the presence/absence of each hormone deficiency, and the presence/absence of any mental disorder. Consecutive variables were divided into two groups statistically. The age at time of survey was divided into under or over $50 \mathrm{yrs}$, the age at time of the diagnosis of hypoadrenalism was under or over $20 \mathrm{yrs}$, and the duration of steroid replacement was under or over 4 yrs. The lower the AIC score, the more information the explanatory variable gave to the objective variable. Statistically, the lower the AIC, the better the model; moreover, it was more explanatory when plural items overlapped. Therefore, the items that could reduce the AIC were regareded as effective characteristics for the clinical risk of adrenal crisis.

We estimated the relative risk of the risk factors with a Cox palindromic analysis. We also examined the difference in frequency by Fisher's exact test.

\section{Results}

The age distribution at time of survey is shown in Fig. 1 and the classification of explanatory variables is shown in Table 2. The age at time of survey was from 16 to 86 yrs old (average $47.4 \mathrm{yrs}$ ), the age at time of diagnosis of hypoadrenalism was from 5 to 71 yrs old (average $24 \mathrm{yrs}$ ). The number of patients with secondary adrenal insufficiency was larger than those with primary insufficiency, and there was no difference in gender. As to steroid hormone replacement therapy, those receiving $\mathrm{G}+\mathrm{M}$ were in the majority, and only 7 patients were receiving $\mathrm{G}$ alone. There were 88 patients of GH deficiency and 53 patients with sex hormone deficiency. The number of patients of diabetes insipidus was 48 . There were 10 cases of mental disorders, consisting of depression $(\mathrm{N}=5)$, poor intelligence $(\mathrm{N}=1)$, procephalic impairment $(\mathrm{N}=3)$, and dementia after surgery $(\mathrm{N}=1)$.

Forty $(29 \%)$ of the 137 patients had at least one episode of adrenal crisis, $6(27 \%)$ of the 22 primary hypoadrenalism cases and 34 (30\%) of the 115 secondary hypoadrenalism cases with no difference between 


\section{Primary adrenal insufficiency ( $\mathrm{N}=\mathbf{2 2}$ )}

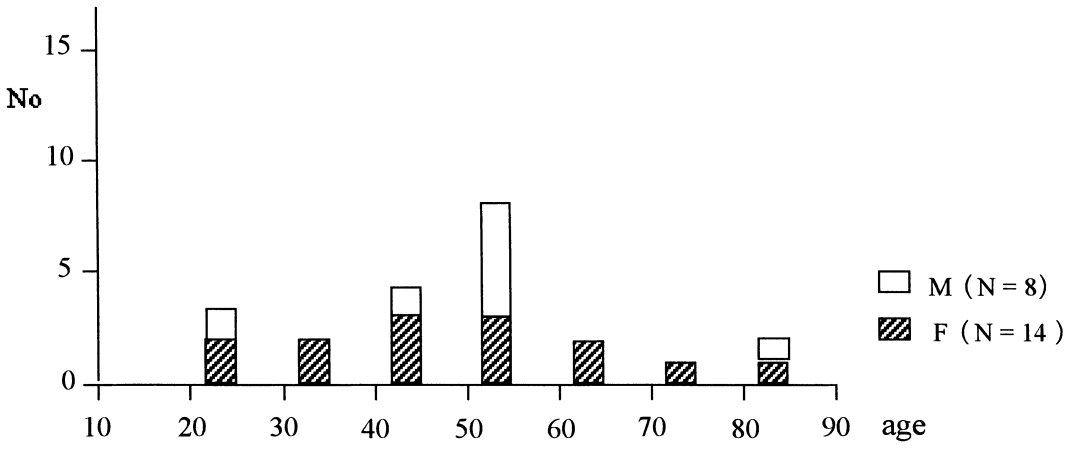

\section{Secondary adrenal insufficiency ( $N=115$ )}

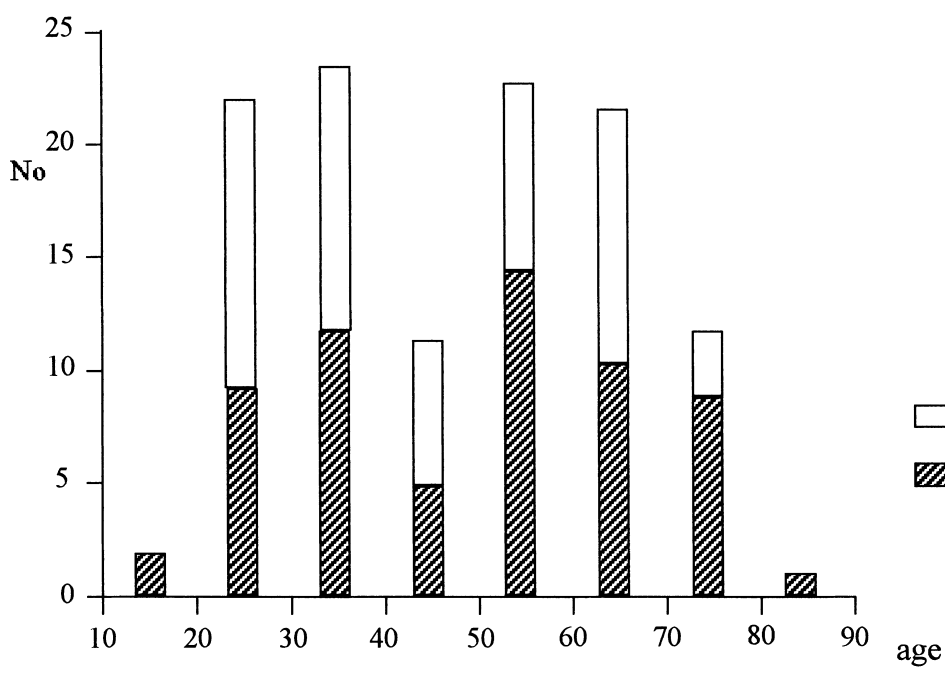

Fig. 1. Age distribution at the time of survey

Table 2. Classification of explanatory variables

\begin{tabular}{|c|c|c|c|c|}
\hline Explanatory variables & & No & Adrenal crisis $(+)$ & Adrenal crisis (-) \\
\hline \multirow[t]{2}{*}{ Gender } & Male & 60 & 14 & 46 \\
\hline & Female & 77 & 26 & 51 \\
\hline \multirow[t]{2}{*}{ Disease classification } & Primary & 22 & 6 & 16 \\
\hline & Secondary & 115 & 34 & 81 \\
\hline \multirow[t]{2}{*}{ Steroid hormone } & $\mathrm{G}+\mathrm{M}$ & 130 & 38 & 92 \\
\hline & $\mathrm{M}$ & 7 & 2 & 5 \\
\hline \multirow[t]{2}{*}{ GH deficiency } & + & 88 & 28 & 60 \\
\hline & - & 49 & 12 & 37 \\
\hline \multirow[t]{2}{*}{ Sex hormone deficiency } & + & 53 & 24 & 29 \\
\hline & - & 84 & 16 & 68 \\
\hline \multirow[t]{2}{*}{ Diabetes insipidus } & + & 48 & 8 & 40 \\
\hline & - & 89 & 32 & 57 \\
\hline \multirow[t]{2}{*}{ Mental disorder } & + & 10 & 8 & 2 \\
\hline & - & 127 & 32 & 95 \\
\hline \multirow[t]{2}{*}{ Age } & $<50$ & 68 & 14 & 54 \\
\hline & $\geq 50$ & 69 & 26 & 43 \\
\hline \multirow[t]{2}{*}{ Age at diagnosis } & $<20$ & 41 & 6 & 35 \\
\hline & $\geq 20$ & 96 & 34 & 62 \\
\hline \multirow[t]{2}{*}{ Duration of steroid replacement } & $<4$ & 24 & 2 & 22 \\
\hline & $\geq 4$ & 113 & 38 & 75 \\
\hline
\end{tabular}


the two groups. Fig. 2 shows the age distribution at the time of crisis. Eleven patients had suffered an adrenal crisis more than once.

Table 3 shows the AIC score. Steroid replacement of more than 4 yrs in duration contributed most to the occurrence of adrenal crisis, followed by mental disorder, sex steroid deficiency, and an age of more than 20 yrs at time of diagnosis of adrenal insufficiency. The distribution of the steroid replacement period is shown in Fig. 3. As regards an adrenal crisis, 1 (4\%) of the 24 had received less than $4 \mathrm{yrs}$ of steroid replacement therapy, and $39(35 \%)$ of the 113 were over 4 yrs. In patients with a mental disorder, eight $(80 \%)$ of the 10 suffered an adrenal crisis. With regard to sex hormone deficiency, there were 52 patients with untreated hypogonadism of whom $24(46 \%)$ had one or more episode of adrenal crisis. Regarding the combination of plural explanatory variables, the AIC of the combination of more than 4 yrs' duration of steroid replacement and mental disorder was -18.82 , and that of more than 4 yrs' duration of steroid replacement and sex hormone deficiency was -16.93 . Furthermore, the AIC of the combination of steroid replacement therapy of more than 4 years' duration, mental disorder and an age of older than 20 yrs old at the time of diagnosis of hypoadrenalism was -21.63 , and that of steroid replacement therapy of more than 4 yrs' duration, mental disorder and sex hormone deficiency was -21.22 . Thus, the values of the AIC were lower in plural combinations, which were shown to have an effective synergy. On the other hand, the class of adrenocortical hormone replaced, the gender, any GH deficiency and the etiology of hypoadrenalism contributed little to the occurrence of adrenal crisis.

We further examined the relationship between hypogonadism and adrenal crisis. As patients with hypogonadism in the primary adrenal insufficiency group were all elderly postmenopausal women, it seemed that their hypogonadism was due to aging. There were 46 patients (10 males and 36 females) with untreated hypogonadism due to hypothalamo-hypophysial impairment out of the 115 patients with secondary adrenal insufficiency, and 22 (48\%) of them had suffered an adrenal crisis.

We next applied the AIC in the analysis of the risk of adrenal crisis in patients with secondary adrenal in-

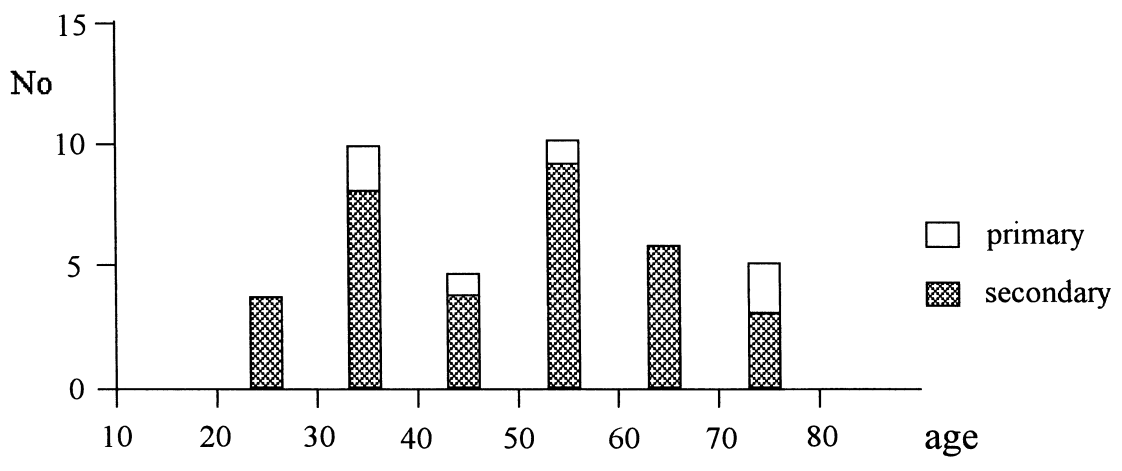

Fig. 2. Age distribution at the time of adrenal crisis

Table 3. AIC in adrenal insufficiency

\begin{tabular}{lcccc}
\hline \multicolumn{1}{c}{ Explanatory variables } & AIC & Adrenal crisis $(+)$ & Adrenal crisis $(-)$ & $\mathrm{p}$ \\
\hline 1. Duration of steroid replacement $(\geq 4$ yrs) & -11.08 & 39 & 74 & 0.0023 \\
2. Mental disorder (+) & -10.08 & 8 & 2 & 0.0008 \\
3. Sex hormone deficiency (+) & -9.47 & 24 & 28 & 0.0009 \\
4. Age at diagnosis of adrenal insufficiency $(\geq 20$ yrs) & -4.54 & 34 & 62 & 0.0145 \\
5. Diabetes insipidus (+) & -3.95 & 8 & 40 & 0.019 \\
6. Age ( $\geq 50$ yrs) & -2.9 & 26 & 43 & 0.0383 \\
7. Steroid hormone (G) & -0.92 & 2 & 5 & $>0.999$ \\
8. Sex (female) & 0.2 & 26 & 51 & 0.1922 \\
9. GH deficiency (+) & 0.95 & 28 & 60 & 0.4352 \\
10. Disease classification (secondary) & 1.87 & 34 & 81 & 0.8068 \\
\hline
\end{tabular}




\section{Primary adrenal insufficiency ( $\mathrm{N}=\mathbf{2 2}$ )}

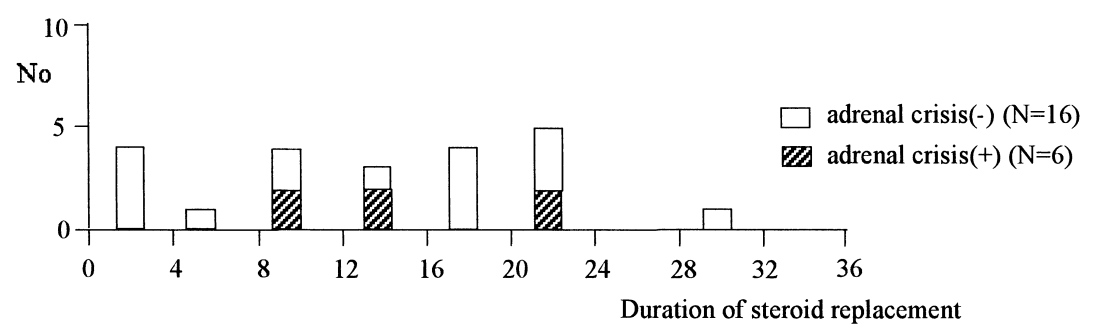

Secondary adrenal insufficiency ( $N=115$ )

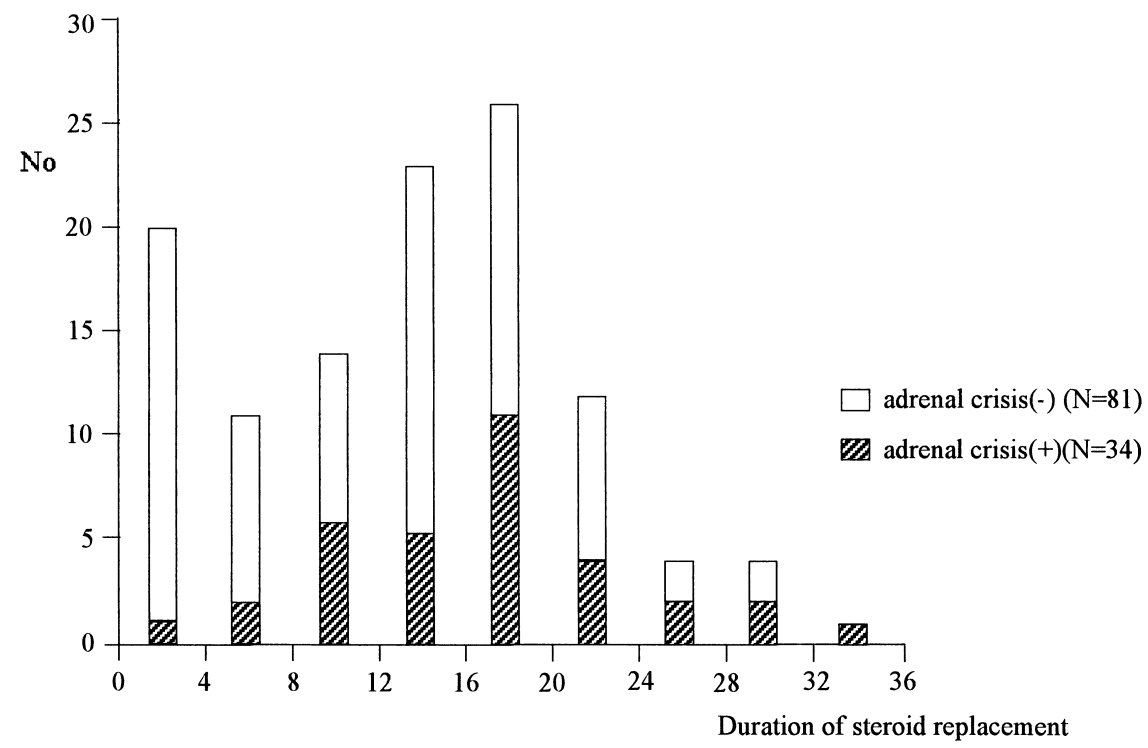

Fig. 3. Distribution of duration of steroid replacement

Table 4. AIC in patients with secondary adrenal insufficiency

\begin{tabular}{lrccc}
\hline \multicolumn{1}{c}{ Explanatory variables } & AIC & Adrenal crisis $(+)$ & Adrenal crisis $(-)$ & $\mathrm{p}$ \\
\hline 1. Sex hormone deficiency $(+)$ & -8.84 & 22 & 24 & 0.0013 \\
2. Duration of steroid replacement $(\geq 4$ yrs) & -7.15 & 33 & 62 & 0.0412 \\
3. Mental disorder (+) & -5.54 & 7 & 2 & 0.0026 \\
4. Diabetes insipidus (+) & -5.18 & 8 & 40 & 0.019 \\
5. Age at diagnosis of adrenal insufficiency $(\geq 20$ yrs) & -4.36 & 29 & 51 & 0.0368 \\
6. Steroid hormone (G) & -3.66 & 2 & 1 & 0.204 \\
7. Age ( $\geq 50$ yrs) & -2.76 & 22 & 34 & 0.0366 \\
8. Sex (female) & -1.56 & 23 & 40 & 0.0926 \\
9. GH deficiency $(+)$ & 0.98 & 28 & 60 & 0.4352 \\
\hline
\end{tabular}

sufficiency (Table 4). Sex hormone deficiency was the greatest risk factor with an AIC of -8.84 . In the combination of two explanatory variables, the AIC of the combination of sex hormone deficiency with steroid replacement of more than 4 yrs' duration was
-13.74 , and that of sex hormone deficiency and mental disorder was -12.1 . Considering the combination of three explanatory variables, the AIC of the combination of sex hormone deficiency, steroid replacement of more than 4 yrs' duration and mental disorder was 
-14.48 , and that of sex hormone deficiency, more than 4 yrs' duration of steroid and $G$ replacement was -12.25 .

Next, we investigated the relative risk by multiple logistic palindromic analysis. Patients with untreated hypoganadism $(\mathrm{N}=52)$ had a significantly higher relative risk of 3.70 (95\% confidential interval: 1.75 7.98) compared to those without hypogonadism $(\mathrm{N}=$ $15)$, or with treated hypogonadism $(\mathrm{N}=70)$. Furthermore, we examined the rate of sideration of adrenal crises among patients younger than 50 yrs old with hypogonadism to exclude hypogonadism by aging. Patients treated with sex hormones (5/51: 10\%) suffered a lower frequency of adrenal crisis than those who remained untreated $(7 / 11: 64 \%, p=0.0004)$.

\section{Discussion}

The diagnosis of adrenal crisis must be treated as an emergency, and is based on physical symptoms and urgent laboratory findings. No consensus has been reached as to the definitive standard of diagnosis. The present study selected the patients diagnosed as undergoing an adrenal crisis by blood pressure, the state of consciousness, serum sodium and serum glucose in addition to the usual symptoms.

Forty (29\%) of the 137 patients had suffered an adrenal crisis. It has been reported that adrenal crises in patients with secondary adrenal insufficiency were fewer than in those with primary adrenal insufficiency [9], but in this study there was no difference between the two types.

We examined the risk factors for an adrenal crisis in patients with chronic adrenal insufficiency based on the analysis of the AIC. As a result, steroid replacement of more than 4 yrs' duration, the presence of a mental disorder, and untreated hypogonadism increased the risk of adrenal crisis. Furthermore, an overlap of these factors raised the risk of adrenal crisis even higher.

A possible reason behind the increased risk of adrenal crisis in patients with steroid replacement therapy of more than 4 yrs' duration was that patients' ability to recognize and to counter stress seemed to declined year by year, and there was moreover an age- and disease progress-related deterioration in both physical and psychological adaptability. It was difficult to elucidate it in this study, but it was postulated that patients under a medication regimen of longer than 4 years possibly require re-education.

Mental disorders in the present study included depression, poor intelligence, procephalic impairment and dementia after surgery. Many patients with a mental disorder had suffered an adrenal crisis. Moreover, four of them had repeated adrenal crises. It was suggested that a support system that includes family knowledge and assistance is important for prevention of adrenal crisis.

There is no report of the effect of hypogonadism on adrenal crisis. However, it has been reported that testosterone and estrogen play an important role in the functioning of brain cognition and preservation of memory [10-12], and also that improvement in physical and mental activities were obtained by sex hormone replacement $[13,14]$. There are several reports that the functional deterioration of the ovary increases the release of the pro-inflammatory cytokines IL-1, IL6 , TNF- $\alpha$ [15]. Therefore, it is suggested that hypogonadism is a trigger of infection that could cause an adrenal crisis. Moreover, the levels of DHEA-S and 17-KS seemed to be important for hypogonadism, but we could not examine these factors due to the lack of available data.

In summary, the present study clarified that longterm glucocorticoid therapy, the presence of a mental disorder, and untreated hypogonadism were high risk factors in inducing an adrenal crisis, and that there was an overlapping effective of these factors. Sex hormone replacement therapy was suggested to be able to reduce the incidence of adrenal crisis.

\section{Acknowledgments}

We greatly appreciate the assistance of Dr. Naomi Hizuka, Tokyo Women's Medical University, for the diagnoses of GH deficiency. 


\section{References}

1. Yamaji T, Ishibashi M (1985) Long-term prognosis of patients with adrenal insufficiency. In: Annual Report of the Ministry and Welfare "Disorders of adrenal hormones" Research Committee 145-152 (In Japanese).

2. Yamaji T, Ishibashi M (1995) Long-term prognosis of patients with secondary adrenal insufficiency. In: Annual Report of the Ministry and Welfare "Diencephalohypophysial disorders" Research Committee 171-174 (In Japanese).

3. Braatvedt GD, Newrick PG, Corrall RJ (1990) Patients' self administration of hydrocortisone. BMJ 301: 1312.

4. Flemming TG, Kristensen LO (1999) Quality of selfcare inpatients on replace-ment therapy with hydrocortisone. J Intern Med 246: 497-501.

5. Peacey SR, Pope KS, Naik RD (1993) Corticosteroid therapy and intercurrent illness: the need for continuing patient education. Postgrad Med J 69: 282-284.

6. Consensus guideline for the diagnosis and treatment of adult growth hormone deficiency (1998): Summary statement of the Growth Hormone Research Society workshop on adult growth hormone deficiency. J Clin Endocrinol Metab 83: 379.

7. Akaike H (1973) Information theory and an extension of the maximum likelihood principle. In: Petrov BN, Csaki F (eds) 2nd International Symposium on Information Theory, Akademai Kiado, Budapest, 267-281.

8. Akaike H (1974) A new look at the statistical model identification. IEEE Trans Autosomal Contr 19: 1716-
1723.

9. Teback SP, Dean HJ (1996) Mortality in Canadian children with growth hormone $(\mathrm{GH})$ deficiency receiving GH therapy 1967-1992. The Canadian Growth Hormone Advisory Committee. J Clin Endocrinol Metab 81: 1693-1696.

10. Yaffe K, Lui LY, Zmuda J, Cauley J (2002) Sex hormones and cognitive function in older men. $\mathrm{J} \mathrm{Am}$ Geriatr Soc 50: 707-712.

11. Kirschbaum WOT (2002) Endogeneous estradiol and testosterone levels are associated with cognitive performance in older women and men. Horm Behav 41: 259266.

12. Ohkura T (1998) Sex steroids and brain function. Acta Obst Gynaec Jpn 50: 633-644 (In Japanese).

13. Rudolph I, Zimmermann T, Kaminski K, Jandova K, Borovsky B, Ahrendt HJ, Golbss (2000) Changes in psychic and somatic well-being and cognitive capabilities of peri- and post-menopausal women after the use of a hormone replacement drug containing estradiol valerate and levonorgestrel. Methods Find Exp Clin Pharmacol 22: 51-56.

14. Cohn PF, Cohn JK (1998) Medical and psychological aspects of hormone replacement therapy in postmenopausal women: points of view from a cardiologist and a psychotherapist. Clin Cardiol 21: 875-877.

15. Pfeilschiter J, Koditz R, Pfohl M, Schatz H (2002) Changes in proinflammatory cytokine activity after menopause. Endocr Rev 23: 90-119. 